\title{
Electron diffraction studies of hot molecules. I. Observed and calculated thermal expansions of $\mathrm{SF}_{6}, \mathrm{CF}_{4}$, and $\mathrm{SiF}_{4}$
}

\author{
Steven R. Goates and Lawrence S. Bartell \\ Department of Chemistry, University of Michigan, Ann Arbor, Michigan 48109 \\ (Received 10 February 1982; accepted 30 April 1982) \\ A new method is described for the rapid heating $\left(10^{-5}-10^{-6} \mathrm{~s}\right)$ of gas molecules for study by electron \\ diffraction. Laser irradiation of the tip of a micronozzle makes it possible to determine structures, amplitudes \\ of vibrations, and aspects of anharmonicity of molecules at temperatures much higher than those at which \\ decomposition occurs in conventional oven nozzles. The vibrations and thermal expansions of $\mathbf{S F}_{6}, \mathbf{C F}_{4}$, and \\ $\mathrm{SiF}_{4}$ have been investigated up to 1700,1600 , and $1200 \mathrm{~K}$, respectively. Clear evidence for effects of \\ anharmonicity was observed in amplitudes of vibration as well as mean bond lengths. Various models \\ proposed for the treatment of increases in bond length have been assessed, among which an anharmonic \\ Urey-Bradley field accounted well for results. Comparisons are made with the predictions of Heenan and \\ Robiette based on spectroscopic analyses. The diffraction approach offers a promising method for augmenting \\ spectroscopy in the investigation of intramolecular forces.
}

\section{INTRODUCTION}

In the course of an investigation of laser-pumped $\mathrm{SF}_{6}$ by electron diffration, ${ }^{1,2}$ a number of diffraction patterns were recorded of vibrationally hot molecules that had been excited by accidental irradiation of the nozzle tip. $^{3}$ These patterns, when analyzed, were found to yield information about vibrational anharmonicity that is difficult to derive by spectroscopy. Because the new method of heating offers several striking advantages, we have capitalized upon the accident to develop an effective new procedure for diffraction studies. Gas molecules can be heated from room temperature to well over $1500 \mathrm{~K}$ in the order of a microsecond, thereby permitting investigations of structures and vibrations at temperatures enormously greater than those at which the molecules can long survive without decomposition. Moreover, because such a minute length of the micronozzel is required to be hot, there is no need for special radiation shields of the sort usually found to be essential in high-temperature diffraction studies.

In this paper, we present results for the thermal expansion of covalent bonds and show how they can be interpreted. We extend our previous research ${ }^{3}$ on $\mathrm{SF}_{6}$ to much higher temperatures and report parallel studies of $\mathrm{CF}_{4}$ and $\mathrm{SiF}_{4}$. In the following paper, paper II, ${ }^{4}$ we discuss the seemingly anomalous "shrinkage effects" encountered at high temperatures in the nonbonded distances of these molecules.

\section{EXPERIMENTAL}

Molecules issuing from a heated nozzle were examined by electron diffraction. The attainment of high temperatures was accomplished by focusing the $\mathrm{cw}$ output of a $40 \mathrm{~W}$ infrared $\mathrm{CO}_{2}$ laser onto the nozzle near its tip. A section of a platinum hypodermic needle, $10 \mathrm{~mm}$ long and $0.2 \mathrm{~mm}$ in diameter, served as the nozzle barrel. The experimental arrangment was the same as that described in Refs. 1 and 2. Diffraction patterns were recorded on $4 \times 5$ in. Kodak electron image plates. All exposures were taken through an $r^{3}$ sector from a camera distance of $21 \mathrm{~cm}$ with an electron accelerating voltage of $40 \mathrm{kV}$. Data lie in the range
$4.0 \AA^{-1}<s<21.1 \AA^{-1}$. The use of a single camera distance, with its limited $s$ range, was justified by the impracticability of reproducing nozzle temperatures from run to run. ${ }^{5}$ Exposures were taken in sets of five, each set consisting of a "blank" plate taken with the laser on, but no sample flowing, to record extraneous exposure, three hot-sample plates with the laser on, and a "control" plate with the laser off.

Sulfur hexafluoride with a stated purity of $99.99 \%$ was obtained from M. G. Scientific Co.; carbon tetrafluoride with a stated minimum purity of $99.7 \%$, from Linde Specialty Gases, and silicon tetrafluoride with a stated minimum purity of $99.6 \%$, from Matheson. Analysis by mass spectroscopy confirmed adequate purity in the chemicals as they were received. Vapor pressures in the sample introduction system were controlled by immersing the sample reservoir in an appropriate slush bath. Reservoir pressures in the experiments were between 70-170 Torr for $\mathrm{SF}_{6}, 60-90$ Torr for $\mathrm{CF}_{4}$, and 120-195 Torr for $\mathrm{SiF}_{4}$. Experimental conditions for individual plates are available as supplemental material. ${ }^{6}$ A liquid nitrogen trap inside the diffraction chamber effectively condensed $\mathrm{SF}_{6}$ and $\mathrm{SiF}_{4}$. It was ineffective in trapping $\mathrm{CF}_{4}$ for which chamber pressures rose to values between $10^{-4}$ and $5 \times 10^{-4} \mathrm{Torr}$, much above normal sample background pressures of about $5 \times 10^{-6}$ Torr. In the course of experiments some decomposition of $\mathrm{SiF}_{4}$ was observed. As discussed in Ref. 5, it was believed to have no effect on the results obtained.

\section{ANALYSIS OF DATA}

\section{A. Determination of diffraction intensities}

Out of 45 plates including blanks taken of $\mathrm{SF}_{6}, 60$ of $\mathrm{CF}_{4}$, and 60 of $\mathrm{SiF}_{4}, 38,50$, and 43 plates were judged to be of sufficient quality for analysis. A modified Sinclair-Smith recording microphotometer was employed to measure optical transmission across each plate while the plate was spun to average over emulsion grains. Transmittances were converted into absorbances $A$, and absorbances were corrected for nonlinear emulsion response via Eq. $(1)^{7}$ to obtain exposures $E$ : 


$$
E=A\left(1+\alpha_{1} A+a_{2} A^{2}+\alpha_{3} A^{3}+\cdots\right) .
$$

Constants $\alpha_{1}=0.116, \alpha_{2}=0.0179$, and $\alpha_{3}=0.00312$ were used in the analysis of the first $18 \mathrm{SF}_{6}$ plates and all $C F_{4}$ plates, and $\alpha_{1}=0.1695, \alpha_{2}=0.0383, \alpha_{3}$ $=0.00974, \alpha_{4}=0.00264$, and $\alpha_{5}=0.000746$ were used in the analysis of the last $12 \mathrm{SF}_{6}$ plates and all $\mathrm{SiF}_{4}$ plates. It was discovered during the workup of the data for $\mathrm{SiF}_{4}$ that the first set of emulsion calibration constants was nonoptimum. A new calibration procedure ${ }^{5}$ was devised to obtain the second set of constants. It was found, however, that changes in derived molecular parameters with increasing vibrational excitation are little affected by the choice of the sets of emulsion calibration constants, when the absorbances of hot sample plates and control plates lie within a few tenths of $a$ unit to each other. ${ }^{y}$ Experimental intensities of representative diffraction plates are available as supplementary material. 8

\section{B. Structure analysis}

Mean-square amplitudes of vibration and mean-internuclear distances were determined from diffraction intensities by a standard least-squares procedure. ${ }^{9}$ Theoretical intensities were calculated using the tabulation of revised ${ }^{10}$ elastic scattering factors of Schafer, Yates, and Bonham ${ }^{11}$ and the inelastic factors of Tavard et al. ${ }^{12}$ Experimental intensities were corrected for intramolecular dynamic scattering and for an average of nonrandom control plate residuals $\overline{\Delta M}_{r}(s)$ as described and justified in Refs. 1 and 2.

Theoretical dynamic scattering intensities for various temperatures were calculated for the present work with the scheme of Miller and Bartell. ${ }^{13}$ An attempt was made to compensate $\overline{\Delta M}_{r}$ for temperature effects by multiplying the $298 \mathrm{~K}$ function by $\exp \left\{-\left[l^{2}(T)-l^{2}(298)\right] s^{2} /\right.$ $2\}$, where $l^{2}$ is the mean-square bond amplitude. The choice of asymmetry parameters is important to the derivation of internuclear distances by electron diffraction. 14,15 Temperature-dependent asymmetry parameters $\hat{a}^{16}$ were calculated from a model published elsewhere ${ }^{15}$ and are shown in Fig. 1(a), 1(b), and 1(c).

For reasons that will be apparent in paper $\mathrm{II}^{4}$ dis tances between bonded and nonbonded atom pairs in all three molecules were refined separately without the imposition of a self-consistent molecular geometry. In the case of $\mathrm{SF}_{6}$, however, this practice led to unreasonable (negative) nonbonded shrinkages ${ }^{17}$ at room temperature. The $\overline{\Delta M}_{r}(s)$ correction for $\mathrm{SF}_{8}$ was determined, therefore, from refinements of control plates in which a self-consistent geometry was imposed with Cyvin's ${ }^{17}$ theoretical shrinkages of 0.00063 and $0.00262 \AA$ respectively for the cis and trans distances. A similar procedure was unnecessary for $\mathrm{CF}_{4}$ and $\mathrm{SiF}_{4}$. Background functions (quintic polynomial plus exponential term for $\mathrm{SF}_{6}$ and $\mathrm{SiF}_{4}$ and quartic polynomial plus exponential term for $\mathrm{CF}_{4}$ ) were refined simultaneously with molecular parameters. ${ }^{8}$ While both $\mathrm{dy}-$ namic scattering and $\overline{\Delta M}_{r}(s)$ corrections were applied to $\mathrm{SF}_{8}$ experimental intensities, only dynamic scattering corrections were applied to $\mathrm{CF}_{4}$ intensities. $A$ suitable $\overline{\Delta M}_{r}(s)$ correction applicable to all $\mathrm{CF}_{4}$ plates

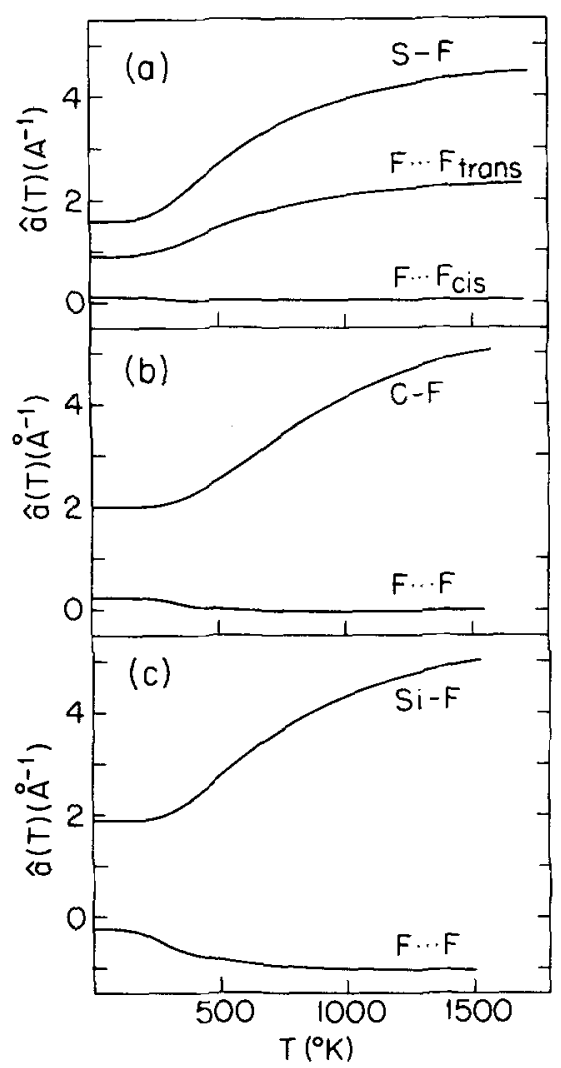

FIG. 1. Temperature dependence of asymmetry parameters $\hat{a}$ for each type of atom pair in (a) $\mathrm{SF}_{6}$, (b) $\mathrm{CF}_{4}$, and (c) $\mathrm{SiF}_{4}$ calculated according to the procedure of Ref. 15.

could not be determined, even though individual residuals seemed to show high point-to-point correlations, because the residuals were not reproducible from plate to plate. This was apparently due to a dependence of the residuals upon the background pressure of $\mathrm{CF}_{4}$ in the diffraction chamber. In the case of $\mathrm{SiF}_{4}$, calculated dynamic scattering corrections were so small that the only $\overline{\Delta M}_{r}(s)$ corrections were used. In all three cases it was found however that neither dynamic scattering nor $\overline{\Delta M_{r}}(s)$ corrections had an important effect on the determination of changes in molecular parameters caused by heating the samples. The primary purpose for applying the corrections, as discussed in Refs. 1 and 2 , is to estimate uncertainties from random noise in the data. While residuals from fits of corrected room temperature data show little point-to-point correlation and reproducibility between plates, increasingly nonrandom residuals are evident for fits of intensities at increasingly higher temperatures. This increasing nonrandomness is due at least in part to a nonoptimum computation of parameter $\hat{a}$ for nonbonded distances as discussed in paper II. ${ }^{4}$

\section{Inference of temperature}

Temperatures could be inferred in our work only from measurements of the amplitudes of molecular vibrations. To each internuclear distance in a given molecule a temperature can be assigned by comparing measured amplitudes with amplitudes calculated for various tem- 
peratures. In order to compensate roughly for systematic errors, arising largely because of the small scattering angles and limited range over which our data were collected, experimental mean-square amplitudes $l_{\&}^{2}$ were adjusted by small additive constants to force experimental amplitudes to agree with calculated amplitudes at room temperature. Amplitudes were calculated by a normal coordinate treatment ${ }^{18}$ using force constants taken from Weinstock and Goodman ${ }^{19}$ for $\mathrm{SF}_{8}$, from Schimanouchi et al. ${ }^{20}$ for $\mathrm{CF}_{4}$, and from Clark and Rippon ${ }^{21}$ for $\mathrm{SiF}_{4}$. Neglect of anharmonicity in the normal coordinate treatment, however, leads to an increasing systematic error in the deduction of temperature increases. A rough estimation of the influence of anharmonicity upon bond amplitudes can be made by treating the bonds as diatomic oscillators and using the formulas derived by Kuchitsu ${ }^{22}$ for the amplitude of vibration of a Morse diatomic oscillator. Such rough estimations were used to augment the normal coordinate bond amplitudes for anharmonicity, and these augmented amplitudes were employed in the inference of temperature. Temperatures derived from the mean-square bond amplitudes were used to update asymmetry constants $\hat{a}$ and corrections to intensity for each successive refinement.

\section{Error analysis}

Error limits applicable to thermal shifts in parameters of $\mathrm{SF}_{6}$ and $\mathrm{SiF}_{4}$ were calculated from residuals in the "corrected intensities" by application of standard random-error theory. ${ }^{23}$ An extensive discussion of the approach is given in Refs. 1 and 2. For diffraction patterns taken at modest temperatures, this procedure appears to be valid and uncertainties inferred from least-squares analyses of a given pattern correlate well with the scatter in parameters derived from separate patterns. At higher temperatures corrected residuals became decidedly less random, in part because the shapes of the nonbonded radial distribution functions were poorly modeled by the assumed $\hat{a}$ parameters. This almost certainly has a greater effect on the nonbonded internuclear distances derived than on the amlitudes of vibration for the bonded distances that are of concern in this paper. Because of this non-randomness of residuals, the application of random error theory to high-temperature analysis is of limited validity. The same is true for $\mathrm{CF}_{4}$ analyses at all temperatures, because of the impossibility of introducing $\overline{\Delta M}_{r}(s)$ corrections.

Uncertainties in estimating temperatures arise from another problem associated with the harmonic form of the force field adopted in the normal coordinate analyses. This will be discussed in a later section.

\section{E. Influence of background pressure}

Delocalization of sample in the diffraction chamber blurs the diffraction pattern and can lead to significant errors in the deduction of mean internuclear distances. ${ }^{24}$ In the investigations of $\mathrm{SF}_{8}$ and $\mathrm{SiF}_{4}$ this was not a problem, but inefficient trapping of the highly volatile gas $\mathrm{CF}_{4}$ resulted in relatively high background pressures

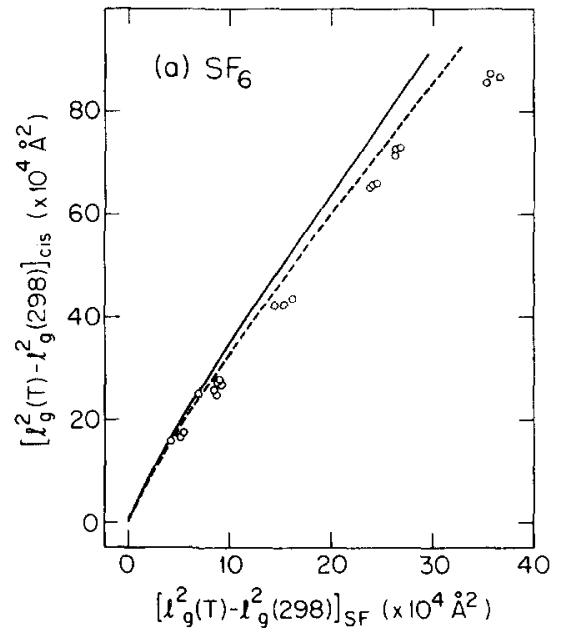

FIG. 2. Thermally induced increase in mean-square $F F_{\text {ors }}$ amplitude vs corresponding increase in mean-square SF amplitude in $\mathrm{SF}_{6}, 300 \mathrm{~K}<T<1700 \mathrm{~K}$. Solid line represents normal coordinate calculations based on harmonic force field. Dashed line corresponds to corrections treating bonds as Morse oscillators, after Kuchitsu, Ref. 22. Circles represent experiment.

during the recording of diffraction patterns for this molecule. Scattering from background molecules, shift ing the effective camera distance, may account for the previously noted dependence of $\mathrm{CF}_{4}$ residuals on background pressure. An attempt to estimate the effect upon the derived $r_{e}$ values of the scattering from background molecules was made some years ago. ${ }^{24}$ Corrections calculated with this rough model reduced scatter in our data slightly, but did not affect the mean dependence of bond distance on bond amplitude observed in the data (see Fig. 7).

\section{RESULTS}

The most conspicuous effect of heating the molecules is a marked increase in the amplitudes of vibration. Although no independent measurements of gas temperature were possible in our study to test the correspondence of experimental amplitudes of vibration to theoretical amplitudes, internal consistency could be checked by comparing amplitudes for different internuclear distances in the same molecule with each other, as shown in Figs. 2-5.

Another effect of heating the gas nozzle is a characteristic diminution of intensity of scattered electrons as the temperature of the gas in the nozzle increases, ${ }^{1.25}$ following a $T^{-0.8}$ law, approximately, in the present work.

Finally, as demonstrated in Figs. 6-9, a significant increase in mean bond length $r_{g}$ with increasing temperature occurs for all three fluorides. For comparison, the data of Kelley and $\mathrm{Fink}^{28}$ for $\mathrm{SF}_{8}$ up to $500 \mathrm{~K}$ are also included in Fig. 6. The increases in bond lengths calculated by various methods discussed in the following section are also shown. 


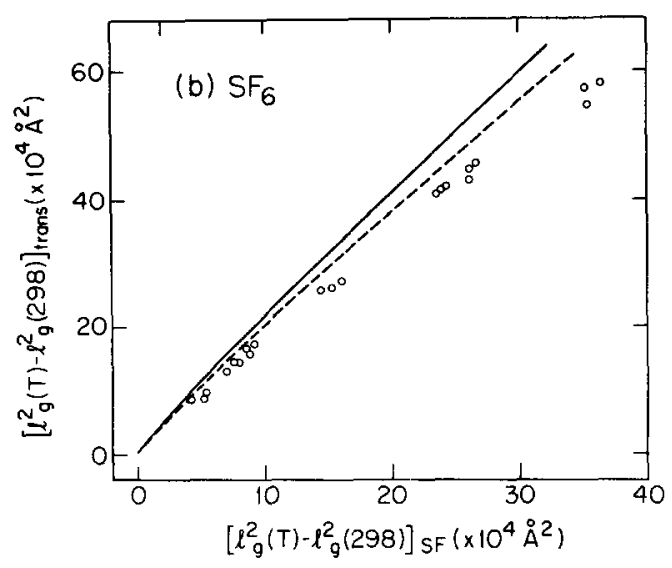

FIG. 3. Increase in mean-square $F F_{\text {trens }}$ amplitude vs increase in mean-square $\mathrm{SF}$ amplitude in $\mathrm{SF}_{6}, 300 \mathrm{~K}<T<1700 \mathrm{~K}$. See the caption for Fig. 2 .

\section{DISCUSSION}

\section{A. Effect of anharmonicity on amplitudes and inferences of temperature}

For infinitesimal vibrations the mean-square amplitudes of vibration depend upon the quadratic force constants. An enormous literature exists ${ }^{27}$ in which the assumption is made that anharmonic contributions to the amplitudes of vibration can be neglected. In the present work, even if the nozzle tip temperatures had been measured, the rapid heating $\left(\sim 10^{-6} \mathrm{~s}\right)$ precludes a direct measure of the vibrational temperatures achieved and we are compelled to resort to normal coordinate theory (harmonic force field) to get a first estimate of temperatures. Fortunately, in a recent study Kelley and $\mathrm{Fink}^{26}$ measured the amplitudes of vibration in $\mathrm{SF}_{6}$ as a function of known gas temperature in experiments with an effusive nozzle, under conditions where molecular vibrations and nozzle can be expected to remain in thermal equilibrium. Changes in mean-square amplitudes of vibration observed by Kelley and Fink for temperatures up to $500 \mathrm{~K}$ correspond well with those from normal coordinate calculations. In our experiments, however, temperatures considerably exceed $500 \mathrm{~K}$ and,

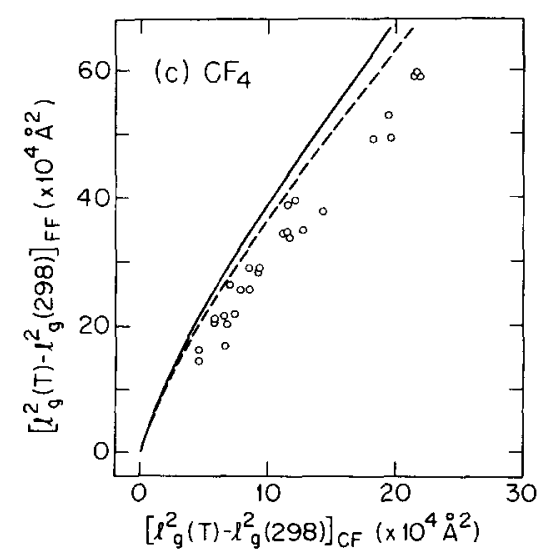

FIG. 4. Increase in mean-square FF amplitude vs increase in mean-square $\mathrm{CF}$ amplitude in $\mathrm{CF}_{4}, 300 \mathrm{~K}<T<1600 \mathrm{~K}$. See the caption for Fig. 2.

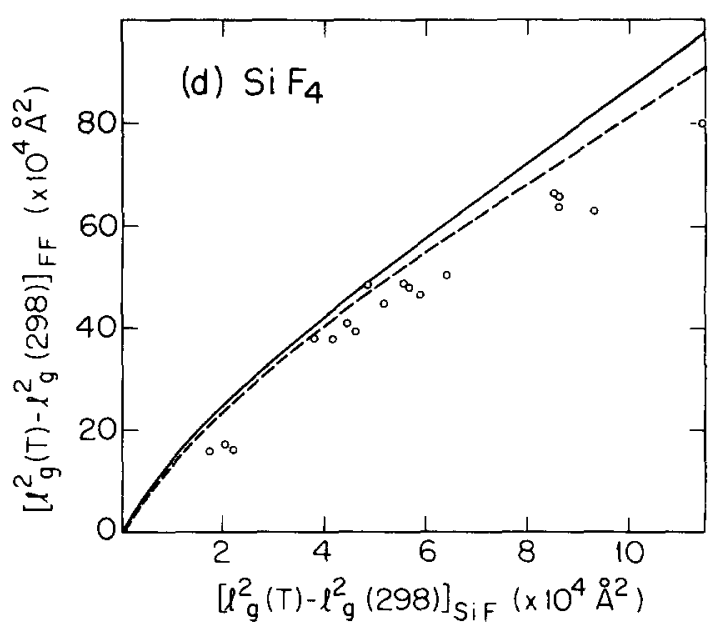

FIG. 5. Increase in mean-square FF amplitude vs increase in mean-square $\mathrm{SiF}$ amplitude in $\mathrm{SiF}_{4}, 300 \mathrm{~K}<T<1200 \mathrm{~K}$. See the caption for Fig. 2.

despite our lack of an independent measure of temperature, we see clear manifestations of anharmonicity upon amplitudes of vibration. These are revealed by plotting changes in mean-square nonbonded amplitudes against changes in mean-square bond amplitudes (Figs. $2-5)$.

At high temperatures, the experimental points for all examples deviate systematically from plots derived from (harmonic) normal coordinate computations. These deviations in the case of the hottest $\mathrm{SF}_{8}$ plates, for example, correspond to differences of nearly $300^{\circ}$ in the temperatures inferred from the bond and from the $c$ is nonbonded amplitudes on the basis of comparison with normal coordinate values. In Sec. III $\mathrm{C}$ was de-

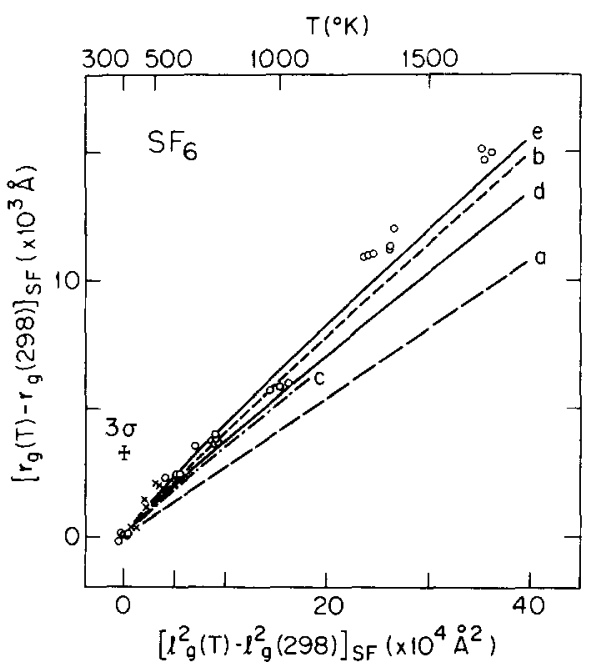

FIG. 6. Thermal expansion of mean bond length in $\mathrm{SF}_{6}$ vs increase in mean-square SF amplitude of vibration. Circles (present work) and crosses (Kelley and Fink, Ref. 26) represent experiment. Curve $a$ calculated according to Eq. (3); curve $b$, Eq. (5); curve $c$ is the prediction of Heenan and Robiette from their spectroscopic force field, Ref. 33; curves $d$ and $e$ are computed from the KBFF formulas of Ref. 35 using constants in sets I and II, respectively, of Table I (see the text). Error bars represent $3 \sigma$ as calculated from corrected room-temperature residuals. 


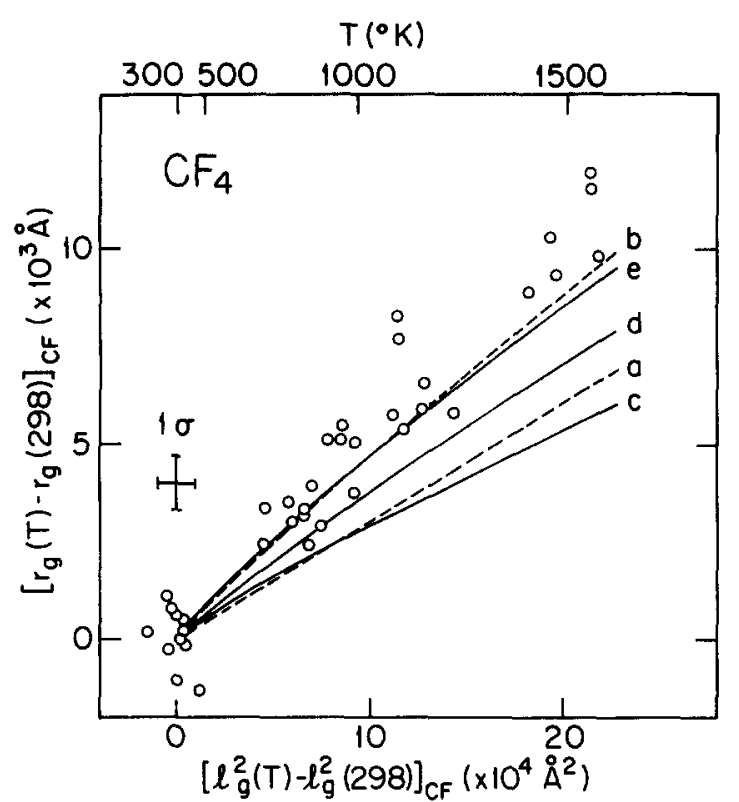

FIG. 7. Expansion of bond length in $\mathrm{CF}_{4}$ vs increase in mean square CF amplitude of vibration. See the caption for Fig. 6 .

scribed a rough estimation of the contribution of anharmonicity to bond amplitudes based on modeling the bonds as Morse diatomic oscillators. This crude augmentation of bond amplitudes does, in fact, significantly improve the correspondence between bond and nonbonded amplitudes, but it accounts for less than half the discrepancy between experiment and normal coordinate theory (see Figs. 2-5). It would not be surprising to find a Morse diatomic model of the bonds to be insufficient; as discussed in a later section and in Ref. 3, such a model does not fully account for increases in mean bond lengths with temperature. On the other hand, it is quite likely that the remaining discrepancy

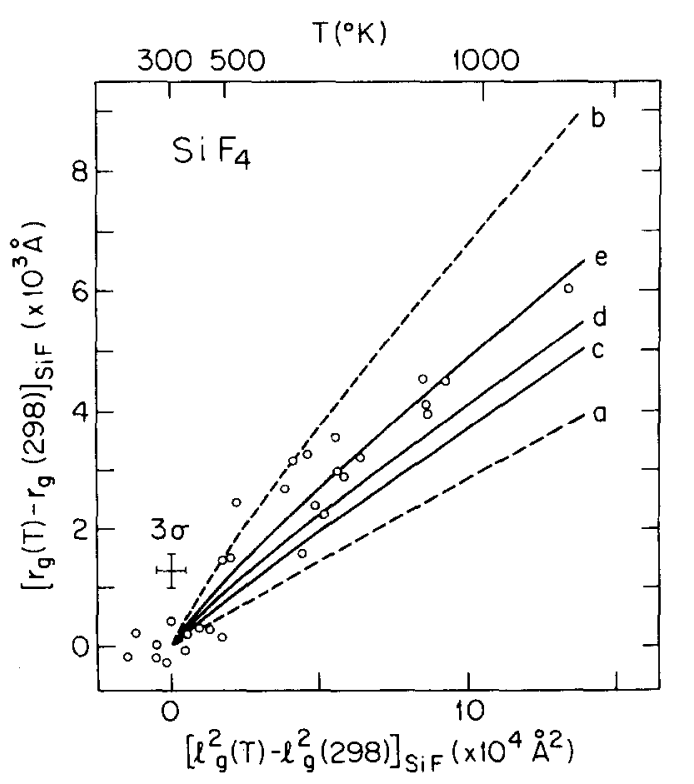

FIG. 8. Expansion of bond length in $\mathrm{SiF}_{4}$ vs increase in mean-square $\mathrm{SiF}$ amplitude of vibration. See the caption for Fig. 6.

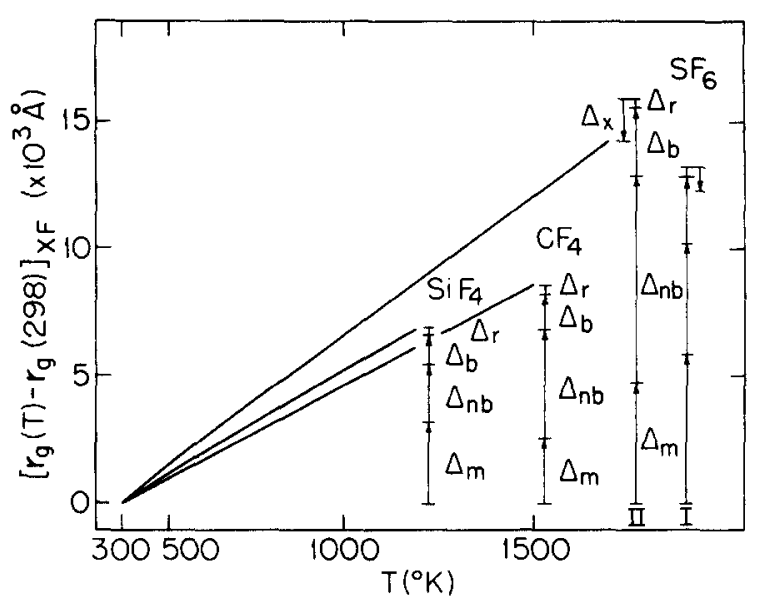

FIG. 9. A comparison of thermal bond lengthenings in $\mathrm{SF}_{6}$, $\mathrm{CF}_{4}$ and $\mathrm{SiF}_{4}$ as calculated with the constants of set II, Table I. Results are decomposed into Morse, nonbonded, bondbending, rotational, and miscellaneous components (see the text). Also shown for comparison are the contributions to $\mathrm{SF}_{6}$ bond lengthenings according to set $\mathrm{I}$.

is due in large measure to the neglected anharmonicity in bending vibrations and its effect on nonbonded amplitudes.

There is a positive aspect to the present method of rapid heating even if it precludes a direct, independent measure of vibrational temperatures. Molecules can be studied at temperatures considerably higher than those at which they can long exist, intact, in conventional oven nozzles. It was reported by Kelley and Fink ${ }^{28}$ that $\mathrm{SF}_{8}$ decomposes at temperatures approximately $700-800^{\circ}$ lower than the maximum temperature at which we studied the molecule.

\section{B. Increases in mean bond length}

Several points of view have been applied to the treatment of thermal expansion in $\mathrm{AX}_{n}$ molecules of the type examined in this work. In the most primitive approach, it is assumed that the force field is purely harmonic in Cartesian displacement coordinates. Hence, atomic vibrational trajectories in bending modes tend to be linear and perpendicular to the mean bond directions. Accordingly, the distances $r_{\alpha}$ between mean atomic positions (leg of a right triangle) are independent of the temperatures while the mean bond lengths $r_{p}$ (mean hypotenuse of a right triangle) increase with vibrational amplitude, if the minor effect of molecular rotation is neglected, as

$$
r_{s}=r_{a}+K
$$

where $r_{\alpha}=r_{\beta}$, the equilibrium bond length and $K$ is $\left[\left\langle(\Delta x)^{2}\right\rangle+\left\langle(\Delta y)^{2}\right\rangle\right] / 2 r_{g}$ in the usual notation. ${ }^{17}$ In diatomic molecules $K$ is zero, of course. Such a model, while sometimes proposed, is a fairy tale implying that stiff bonds stretch in proportion to mean-square amplitudes of the limpest bending modes.

Another view is based on a Morse diatomic oscillator, which is assumed to have a close resemblance to a covalent bond in a polyatomic molecule. For the di- 
atomic case, it has been show $\mathrm{n}^{28}$ that the bond length increases with mean-square amplitude very nearly as

$$
r_{s} \simeq r_{\theta}+3 a l^{2} / 2,
$$

where $a$ is the (temperature independent) Morse asymmetry parameter in the potential energy function. For polyatomic molecules, if the opposite extreme to that in the harmonic model of Eq. (2) is adopted, namely, the assumption that covalent bonds tend to be so stiff that they preserve their lengths in bending motions, it is plausible to apply Eq. (3).

Still another simplified model has been proposed ${ }^{26,29}$ that needs to be evaluated. A rigorous expression for the mean-bond length, again neglecting molecular rotation, is

$$
r_{\varepsilon}=r_{\theta}+K+\langle\Delta z\rangle \text {. }
$$

If the same assumptions are made that entered Eq. (2), namely, that no anharmonic terms enter which tend to preserve bond lengths during bends and that $\langle\Delta z\rangle$ can be replaced by the diatomic correction $3 a l^{2} / 2$, the result is

$$
r_{\varepsilon}=r_{a}+K+3 a l^{2} / 2 \text {. }
$$

Tables of Herschbach and Laurie ${ }^{30}$ provide reasonable values of $a$ for testing Eqs. (3) and (5).

Several authors have shown how to calculate meaninternuclear distances rigorously if the potential energy function is known. ${ }^{31,32}$ Since it is almost never known completely enough to allow the necessary computations to be carried out, however, there is some merit in testing the simpler models introduced above. Our harmonic normal coordinate calculations provide bond amplitudes $l^{2}$ and perpendicular amplitudes $\left\langle(\Delta x)^{2}\right\rangle$ and $\left\langle(\Delta y)^{2}\right\rangle$ of sufficient accuracy for evaluation of the performance of Eqs. (2), (3), and (5).

Not plotted in Figs. 6-8 are the results of applying Eq. (2). This equation, although yielding some thermal expansion, largely for the wrong reason, falls far short of providing enough. Equation (3), the diatomic formula, fares better but, as illustrated, is still insufficient. Marrying Eq. (2) to Eq. (3) to get Eq. (5) can be seen to follow the observed bond expansions of $\mathrm{SF}_{8}$ and $\mathrm{CF}_{4}$ quite closely. It would be injudicious to say that Eq. (5) explains the results, however, because it assembles ad hoc two somewhat contradictory models and neglects several important sources of anharmonicity. That Eq. (5) is not to be trusted, in general, is shown by its poor account of hot $\mathrm{SiF}_{4}$.

Anharmonic force fields as completely established as those of a few triatomic molecules ${ }^{32}$ are not yet available for the molecules discussed in the foregoing. Nevertheless, Heenan and Robiette ${ }^{33}$ have made considerable progress, particularly in the case of $\mathrm{SF}_{8}$, in deriving anharmonic constants from vibrational spectra, although certain potentially significant cubic constants are still unknown. A comparison between thermal increases in bond lengths calculated from spectral data with the present observed increases, then, serves in some measure as a test of the Heenan-Robiette fields. As can be seen in Fig. 6, the Heenan-Robiette predictions for $\mathrm{SF}_{8}$ are fairly good up to the maximum temper- atures they considered. Their less complete fields for $\mathrm{CF}_{4}$ and $\mathrm{SiF}_{4}$ are shown in Figs. 7 and 8 to be less successful in accounting for the full thermal expansions. It appears that inclusion of diffraction data with spectroscopic data would be helpful in the derivation of anharmonic potential constants.

An alternative procedure for treating thermal expansion has been proposed ${ }^{34}$ that is more complete than Eqs. (2), (3), and (5), yet can be applied in the absence of detailed spectroscopic information. It is an anharmonic variant ${ }^{35}$ of a Urey-Bradley type of force field sometimes designated as the KBFF. This field accurately models the quadratic force field, allows naturally for the tendency of bonds to preserve their lengths dur ing bends while incorporating the kinematics of atomic trajectories opposing this tendency. It provides plausible estimates of the essential anharmonic terms. Explicit equations are given in Refs. 3 and 34. In the case of $\mathrm{AB}_{n}$ molecules, the KBFF model leads to an expression for $r_{g}-r_{\theta}$ of simple form, in which contributions to the displacement can be identified with a rotational centrifugal stretch $\Delta_{r}$; a Morse anharmonic stretch $\Delta_{m}$ appropriate for a polyatomic molecule; a stretch $\Delta_{n b}$ formally associated with the increased force of collision between nonbonded atoms as vibrational amplitudes increase; a vibrational centrifugal stretch $\Delta_{b}$ associated with bending modes; and a usually minor residual term $\Delta_{x}$. Results of the present work suggest a somewhat different interpretation of $\Delta_{n b}$.

Force constants required in the calculations are the symmetry force constant $F_{11}$ for the totally symmetric stretch, the KBFF quadratic stretch constant $K$, the $\mathrm{KBFF}$ quadratic bend constant $H$, and the KBFF nonbonded repulsion constants through cubic, $F^{\prime}, F$ and $F_{3}$. Most KBFF constants can be obtained from general valence or symmetry force constants, ${ }^{20,33,35}$ but the nonbonded constants are usually poorly characterized, and direct information about $F_{3}$ in particular is not available. The contribution of nonbonded repulsion to bond lengthening $\Delta_{n b}$ calculated according to the KBFF approach is, however, directly proportional to $F_{3}$ and can be quite large. Various methods exist for estimating the nonbonded constants, including deducing them from the derivatives of an assumed nonbonded potential. ${ }^{34}$ Shimanouchi et al. ${ }^{20,38}$ have proposed a Lennard-Jones 6-12 potential for fluorine-fluorine repulsions from fits of the Urey-Bradley constant $F$ to spectroscopic data for a series of fluorides. Woehler ${ }^{37}$ has determined an exponential form for the nonbonded potential from $a b$ initio and semiempirical calculations. Shown in Figures 6-8 are representative calculations for the lengthening of mean-bond distances in $\mathrm{SF}_{6}, \mathrm{CF}_{4}$, and $\mathrm{SiF}_{4}$. Constants for these calculations are listed in Table I. Quadratic KBFF components were inferred from the published fields of McDowell et al ${ }^{38}$ for $\mathrm{SF}_{6}$ and of Clark and Rippon ${ }^{21}$ for $\mathrm{CF}_{4}$ and $\mathrm{SiF}_{4}$, with the aid of the Woehler nonbonded potential. Values for $K$ and $H$ were deduced from the straightforward relationships between symmetry force constants and KBFF constants ${ }^{4,35}$ and are influenced somewhat by the magnitude of the nonbonded constants. Values for $F^{\prime}, F$ and $F_{3}$ in set $I$ of each entry have been determined by direct 
TABLE I. Constants ${ }^{a}$ for the calculation of thermal expansions of bond lengths according to the method of Ref. 34 .

\begin{tabular}{cccccccccc}
\hline \hline Molecule & Set & $F_{11}$ & $K$ & $H$ & $F^{\prime}$ & $F$ & $F_{3}$ & $r_{\theta}$ & $a$ \\
\hline $\mathrm{SF}_{6}^{b}$ & I & 6.845 & 4.47 & 0.515 & -0.072 & 0.559 & -4.56 & $1.555 \AA$ & $1.6 \AA^{-1}$ \\
& II & 6.845 & 3.63 & 0.251 & -0.125 & 1.03 & -8.51 & $1.555 \AA$ & $1.6 \AA^{-1}$ \\
$\mathrm{CF}_{4}{ }^{\mathrm{c}}$ & I & 9.23 & 5.56 & 0.497 & -0.088 & 0.677 & -5.40 & $1.317 \AA$ & $2.02 \AA^{-1}$ \\
& II & 9.23 & 4.29 & 0.220 & -0.168 & 1.35 & -10.9 & $1.317 \AA$ & $2.02 \AA^{-1}$ \\
$\mathrm{SiF}_{4}{ }^{\mathrm{c}}$ & I & 7.18 & 5.975 & 0.233 & -0.022 & 0.170 & -1.54 & $1.55 \AA^{\circ}$ & $1.9 \AA^{-1}$ \\
& II & 7.18 & 5.88 & 0.177 & -0.033 & 0.318 & -3.02 & $1.55 \AA^{-1}$ & $1.9 \AA^{-1}$ \\
\hline \hline
\end{tabular}

${ }^{2}$ All force constants are in mdyn $/ \AA$. Values of $a$ are taken from Ref. 30 . See the text, Sec. V B for the meaning of sets I and II

${ }^{b}$ Calculated from values in Refs. 37 and 38.

${ }^{\circ}$ Calculated from values in Refs. 21 and 37.

differentiation of the Woehler potential with a slight augmentation from electrostatic contributions, assuming that fluorines each bear 0.15 excess electrons in $\mathrm{SF}_{8}$ and $\mathrm{CF}_{4}$ and 0.20 excess electrons in $\mathrm{SiF}_{4}$. The ratio of nonbonded constants with respect to one another in set II of each table was fixed by the form of the Woehler potential, but the magnitude of the constants was fixed by the spectroscopically determined symmetry constant $F_{34}$, according to the relationship of $F^{\prime}$ and $F$ to $F_{34} \cdot{ }^{39}$ Values of $a$, the Morse asymmetry constant, were taken from the work of Hershbach and Laurie ${ }^{30}$ In calculating $\Delta_{r}$, the rotational centrifugal stretch, we took rotational cooling due to the supersonic nature $e^{2}$ of the gas jet roughly into account by assuming, somewhat arbitrarily, a rotational temperature one-third that of the vibrations; the $\Delta_{r}$ term makes, however, only a small contribution to mean bond length. The force fields of Refs. 20,33, and 36, when cast into the KBFF form and coupled with the Woehler nonbonded potential, give very similar results.

The KBFF description of the fluorides we have studied provides a reasonably good representation of the diffraction results, the constants of set II giving the best fit to observed changes in mean bond lengths in all three cases. As demonstrated previously, ${ }^{3}$ a $6-12$ potential description of the nonbonded repulsion in $\mathrm{SF}_{6}$ used by Person and $\mathrm{Kim}^{40}$ and Shimanouchi etal. ${ }^{36}$ leads to a significant overestimation of bond lengthening in $\mathrm{SF}_{6}$. We have found the same to be true for the 6-12 nonbonded potential of Shimanouchi et al. ${ }^{20}$ for $\mathrm{CF}_{4}$. In the case of $\mathrm{SiF}_{4}$, where fluorine-fluorine separations are larger, the 6-12 potential of Ref. 20 yields nonbonded constants close to those in Table I, set $\mathrm{I}$.

A comparison of the bond lengthening in each of the three fluorides of this study is shown in Fig. 9 along with the various contributions to bond lengthening, according to the KBFF model, calculated from the constants of set II. To illustrate how these calculated contributions are affected by changing the nonbonded potential, the contributions to bond lengthening calculated with the constants of set I are also shown for $\mathrm{SF}_{8}$. Particularly in the case of set II, which gives better agreement, the implied contribution of nonbonded repulsion is surprisingly large. It is so large, in fact, that we were initially inclined to dismiss it as physically im- plausible. Moreover, the magnitude of the Urey-Bradley stretch constant $K$ appears to become unrealistically small as larger nonbonded components are invoked in the framework of the KBFF. As evidence from several sources accumulated, ${ }^{4,41}$ however, the utility of postulating substantial anharmonic contributions from strong "bond-bond repulsions" contributing to the force field mathematically in the same way as do atom-atom interactions in the KBFF, became increasingly attractive. This approach, which simply interrelates a variety of features of theory, structure, and vibrations, will be developed elsewhere.

\section{ACKNOWLEDGMENTS}

This research was supported by the National Science Foundation under Grant No. CHE-7926480. We thank Dr. Richard Heenan, Martin Shetter and William Vance for their advice and considerable assistance. We gratefully acknowledge a generous allocation of computing time from the University of Michigan Computing Center.

${ }^{1}$ L. S. Bartell, M. A. Kacner, and S. R. Goates, J. Chem. Phys. 75, 2730 (1981).

${ }^{2}$ L. S. Bartell, M. A. Kacner, and S. R. Goates, J. Chem. Phys. 75, 2736 (1981).

${ }^{3}$ L. S. Bartell, S. K. Doun, and S. R. Goates, J. Chem. Phys. 70, 4585 (1979).

4S. R. Goates and L. S. Bartell, J. Chem. Phys. 77, 1874 (1982).

${ }^{5}$ S. R. Goates, Ph. D. thesis, University of Michigan, 1981. ${ }^{6}$ See AIP document No. PA PS JCPSA-1866-14 for 14 pages of related materials including conditions under which the diffraction plates were taken, representative intensities and derived results. Order by PAPS number and journal reference from American Institute of Physics, Physics Auxiliary Publication Service, 335 E. 45 St., New York, NY 10017. The price is $\$ 1.50$ for each microfiche (98 pages), or $\$ 5$ for photocopies of up to 30 pages and $\$ 0.15$ for each page over 30 pages. Airmail is additional. Make checks payable to American Institute of Physics.

${ }^{7}$ H. R. Foster, J. Appl. Phys. 41, 5344 (1970); J. F. Hamilton, in The Theory of the Photographing Process, 3rd ed., edited by C. E. R. Mees and T. H. James (Macmillan, New York, 1966).

${ }^{8}$ This is especially true when absorbances are small, as they 
were for the first $18 \mathrm{SF}_{6}$ plates and all $\mathrm{CF}_{4}$ plates.

'L. S. Bartell, D. A. Kohl, B. L. Carroll, and R. M. Gavin, Jr., J. Chem. Phys. 42, 3079 (1965); L. S. Bartell, in Physical Methods of Chemistry 4th ed., edited by A. Weissberger and B. W. Rossiter (Interscience, New York, 1973).

${ }^{10}$ H. L. Sellers, L. Sehafer, and R. A. Bonham, J. Mol. Struct. 49, 125 (1978).

${ }^{11}$ L. Schaffer, A. C. Yates, and R. A. Bonham, J. Chem. Phys. 55, 3055 (1971).

${ }^{12}$ C. Tavard, D. Nicolas, and M. Rouault, J. Chim. Phys. (Paris) 64, 540 (1967).

${ }^{13}$ B. R. Miller and L. S. Bartell, J. Chem. Phys. 72, 800 (1980).

${ }^{14}$ L. S. Bartell, J. Chem. Phys. 23, 1219 (1955); K. Kuchitsu and L. S. Bartell, ibid. 35, 1945 (1961).

${ }^{15}$ L. S. Bartell, J. Mol. Struct. 63, 259 (1980).

${ }^{16}$ Because there is much confusion in the literature about the asymmetry constant used in electron diffraction, we shall designate by $a$ the Morse potential constant, which is independent of temperature, and by $\hat{a}$, the temperature-dependent asymmetry parameter related to the frequency modulation of intensity by the relation $\kappa=\hat{a} l^{4} / 6$.

${ }^{17}$ For a discussion of shrinkage, see S. J. Cyvin, Molecular Vibrations and Mean Square Amplitudes (Elsevier, Amsterdam, 1968), see also Ref. 4.

${ }^{18}$ R. L. Hilderbrandt and J. D. Weiser, J. Chem. Phys. 55, 4648 (1971).

${ }^{19} \mathrm{~B}$. Weinstock and G. Goodman, Adv. Chem. Phys. 9, 170 (1965).

${ }^{20} \mathrm{~T}$. Shimanouchi, I. Nakagawa, J. Hiraishi, and M. Ishii, J. Mol. Spectrosc. 19, 78 (1966).

${ }^{21}$ R. J. H. Clark and D. M. Rippon, J. Mol. Spectrose. 44, 479 (1972).

${ }^{22}$ K. Kuchitsu, Bull. Chem. Soc. Jpn. 40, 498 (1967).

${ }^{23}$ L. S. Bartell and M. G. Anashkin, J. Mol. Struct. 17, 193 (1973).

${ }^{24}$ L. S. Bartell, J. Appl. Phys. 31, 252 (1960).

${ }^{25}$ S. K. Doun, Ph. D. thesis, University of Michigan, 1979.

${ }^{26}$ M. Kelley and M. Fink, Austin Symposium on Molecular
Structure, Austin, Texas, March 3, 1980, J. Chem. Phys. (submitted); M. Fink (private communication).

${ }^{27}$ See, for example, Ref. 17, and the citations therein.

${ }^{28}$ L. S. Bartell, J. Chem. Phys. 23, 1219 (1955).

${ }^{29}$ S. Samdal, D. M. Barnhart, and K. Hedberg, J. Mol. Struct. 35, 67 (1976).

${ }^{30}$ D. R. Herschbach and V. W. Laurie, J. Chem. Phys. 35, 458 (1961). In the case of $\mathrm{SF}_{6}$ a value of $a=1.6 \AA^{-1}$ was adopted, arbitrarily, following Ref. 15, instead of using the Herschbach and Laurie value of $1.8 \AA^{-1}$.

${ }^{31}$ See, for example, K. Kuchitsu and L. S. Bartell, J. Chem. Phys. 36, 2470 (1962); I. M. Mills, J. Phys. Chem. 80, 1187 (1976).

${ }^{32}$ R. L. Hilderbrandt and D. A. Kohl, Theochem. 2, 25 (1981); D. A. Kohl and R. L. Hilderbrandt, ibid. 2, 325 (1981).

${ }^{33}$ R. Heenan, Ph. D. thesis, University of Reading, England, 1979.

${ }^{34}$ L. S. Bartell, J. Chem. Phys. 70, 4581 (1979).

${ }^{35} \mathrm{~L}$. S. Bartell and K. Kuchitsu, J. Chem. Phys, 37, 691 (1962). See also the first reference in Ref. 31.

${ }^{36} \mathrm{~J}$. Hiraishi, I. Nakagawa, and T. Shimanouchi, Spectrochim. Acta 20, 819 (1964).

${ }^{37}$ L. S. Bartell and S. E. Woehler (unpublished). The calculations yielded $149 \exp (-3.75 q)$ for $V_{F F}(q)$ in mdyn $\AA$, where $q$ is the separation of fluorine atoms in $\AA$. A brief description of the calculations is given in Ref. 3 .

${ }^{38}$ R. S. McDowell, J. P. Aldridge, and R. F. Holland, J. Phys. Chem. 80, 1203 (1976).

${ }^{39}$ H. Kim, P. A. Souder, and H. H. Claassen, J. Mol. Spectrosc. 26, 46 (1968).

${ }^{40}$ W. B. Person and K. C. Kim, J. Chem. Phys. 69, 2117 (1978).

${ }^{41}$ See M. J. Rothman, L. S. Bartell, C. S. Ewig, and J. R. Van Wazer, J. Chem. Phys. 73, 375 (1980); L. S. Bartell, A. Gavezzotti, and M. J. Rothman, ibid. 76, 4136 (1982); L. S. Bernstein and M. F. Vernon (unpublished).

${ }^{42}$ In the sense of "Valence-Shell-Electron-Pair-Repulsions," discussed in Ref. 4. 